\title{
Importances Socioculturelles Et Économiques Des Singes Des Fragments De Forêts Sacrées Pour Les Populations Riveraines De Gbétitapéa, Centre-Ouest De La Côte d'Ivoire
}

\author{
Béné Jean-Claude Koffi, \\ Claude-Victorien Kouakou, \\ Kramoko Bamba, \\ Didié Armand Zadou, \\ Université Jean Lorougnon Guédé, Côte d'Ivoire \\ Yamois Venceslas Kouakou, \\ Université Félix Houphouët-Boigny, Côte d'Ivoire
}

Doi:10.19044/esj.2019.v15n36p344 URL:http://dx.doi.org/10.19044/esj.2019.v15n36p344

\section{Résumé}

Les communautés locales, à travers leurs pratiques culturelles, ont conservé des éléments de la nature. Ce mode de gestion de la biodiversité varie en fonction des peuples et des groupes ethniques. Quelle est donc l'importance socioculturelle et économique des fragments forestiers pour les populations locales de Gbétitapéa ? Cette étude vise à montrer l'importance culturelle et économique que revêtent les singes des fragments forestiers pour la population de Gbétitapéa, au centre-ouest de la Côte d'Ivoire. La méthodologie utilisée a consisté en des enquêtes ethnozoologiques auprès des populations riveraines. Au terme de cette étude, il ressort que la majorité des populations connait l'origine sacrée des singes. Bien que sacrés, la viande de singes est consommée par une minorité à Gbétitapéa et en majorité dans les villages voisins. La forêt sacrée et les singes constituent une source économique importante eu égard à l'écotourisme. Une meilleure connaissance des pratiques culturelles pourrait montrer l'importance socioculturelle et économique des sites sacrés afin de prévoir une gestion durable des écosystèmes forestiers.

Mots clés : Gbétitapéa, Singes, Forêt Sacrée, Fragment Forestier 


\title{
Socio-cultural and Economic Importance of Monkeys of Forest Fragments for Local Populations of Gbetitapea, Central west of Côte d'Ivoire
}

\author{
Béné Jean-Claude Koffi, \\ Claude-Victorien Kouakou, \\ Kramoko Bamba, \\ Didié Armand Zadou, \\ Université Jean Lorougnon Guédé, Côte d'Ivoire \\ Yamois Venceslas Kouakou, \\ Université Félix Houphouët-Boigny, Côte d'Ivoire
}

\begin{abstract}
Local communities, through their cultural practices, have preserved elements of nature. This way of managing biodiversity varies according to peoples and ethnic groups. What is the socio-cultural and economic importance of the forest fragments for the local populations of Gbetitapea? This study aims to show the importance of monkeys of forest fragments for the population of Gbetitapéa, in western Côte d'Ivoire, at the cultural and economic levels. The methodology used consisted of surveys of local populations using a mixed approach. At the end of this study, it appears that the majority of the populations knows the sacred origin of the monkeys. Although sacred, monkey meat is eaten by a minority in Gbetitapéa and mostly in neighboring villages. The sacred forest and monkeys are an important economic source for ecotourism. A better knowledge of cultural practices could show the socio-cultural and economic importance of sacred sites in order to plan for sustainable management of ecosystems..
\end{abstract}

Keywords: Gbetitapéa, Monkeys, Sacred Forest, Forest Fragment

\section{Introduction}

Les forêts tropicales subissent d'énormes pressions anthropiques dues à de nombreux facteurs conduisant à la perte de leurs superficies au profit des cultures, à la dégradation de leur biodiversité (Mather, 1992 ; Saunders et al., 1998 ; Altieri et Pengue, 2006 ; FAO, 2006). Au regard de cette dégradation des forêts et de la disparition des espèces animales qu'elles abritent, les surfaces protégées devraient jouer le rôle de refuge pour ces populations 
animales. Cependant, le constat est que ces domaines sont de plus en plus menacés par les pressions anthropiques. Il est donc nécessaire qu'un changement d'approche dans la gestion des ressources naturelles voit le jour (Ndotam, 2005 ; Malan, 2009). Or, l'on sait que les sociétés traditionnelles ont établi depuis longtemps des sites naturels sacrés qu'elles tentent tant bien que mal de préserver de toutes les formes d'agressions anthropiques. Considérées par certains comme de véritables aires protégées, ces sites sacrés ont réellement été reconnues comme pouvant appartenir aux différentes catégories des espaces protégés de l'UICN en 2008 (IUCN, 2008). Parmi ces sites sacrés, les forêts sacrées constituent pour certaines régions marquées par une déforestation massive, les seuls témoins de la végétation originelle existante (Bhagwat et Rutte, 2006).

Ce mode de gestion de la biodiversité varie en fonction des peuples et des groupes ethniques. Dans la plupart des cas, il s'agit d'interdits totémiques dont les raisons sont variables. Par exemple à Soko (au nord-est de la Côte d'Ivoire) et à Gbétitapéa (au centre-ouest de la Côte d'Ivoire), la consommation de la viande de singe constitue un interdit pour les populations qui considèrent cet animal comme leurs aïeux (Kouakou et al., 2017). A Sapia (au centre-ouest de la Côte d'Ivoire), les silures sont interdits à la consommation par les communautés locales qui les considèrent comme responsables de la mort des enfants de l'un de leurs aïeux (Sie et Ibo, 1990). Ces richesses culturelles se retrouvent chez tous les peuples en Afrique (Nikolaus, 2001 ; Adjakpa et Tchabi, 2002 ; Willimas et al., 2014). Pour les communautés riveraines des sites sacrés, l'exploitation des ressources naturelles de ces patrimoines locaux est une question de survie. Leurs moyens d'existence dépendent des ressources biologiques pour se procurer nourriture, combustibles, médicaments, matériel de construction (Zadou, 2011). Aussi, ces sites sacrés assurent-ils une sécurité économique pour les populations locales à travers l'écotourisme (FFEM, 2007). L'importance de ces domaines sacrés pour les communautés riveraines va au-delà des moyens de subsistance. En effet, le maintien de l'identité culturelle de ces communautés dépend de ces patrimoines sacrés et de certains animaux dont la survie dépend de ses sites sacrés. C'est l'exemple de certaines forêts qui ont toujours constitué un sanctuaire pour les populations autochtones (Zadou, 2011).

En Côte d'Ivoire, 6702 forêts sacrées couvrant une superficie globale de 37000 ha ont été recensées entre 1996 et 1998 par Gomé (2003). Cependant, très peu d'études portent sur les relations Homme-faunes de ces fragments de forêts et de leur importance pour les populations locales. Parmi ces forêts sacrées, celle de Gbétitapéa abrite deux espèces de singes sacrés. Quelle importance représentent donc ces singes pour ces populations pour qu'ils bénéficient de leur protection ? Dans cette étude, nous abordons les aspects socioculturel et économique. 


\section{Méthodes}

\subsection{Site d'étude}

La présente étude s'est réalisée dans la région du Haut Sassandra, au centre-ouest de la Côte d'Ivoire. Trois villages situés non loin de la ville de Daloa ont été considérés dans cette étude du fait de leur proximité à la Forêt sacrée de Gbétitatpéa; Ce sont les villages de Gbétitapéa, Zakoua et de Gbalagoua (Figure 1). La région bénéficie d'un climat tropical humide caractérisé par une grande saison de pluie (mi-mars à mi-juillet), une petite saison de pluie (septembre à novembre). Elles alternent avec une grande saison sèche (décembre à mi-mars) et une petite saison sèche (mi-juillet à août). La précipitation moyenne annuelle est comprise entre $1200 \mathrm{~mm}$ et $1600 \mathrm{~mm}$ par an (Brou, 2005 ; Kouamé et al., 2006 ; Koffie et Kra, 2013 ; Norbert et al., 2015). La température moyenne annuelle est de $26^{\circ} \mathrm{C}$ (Koffie et Kra, 2013). La région est sous l'influence du fleuve Sassandra et de ses affluents (le Lobo et le Davo) et du lac du barrage de Buyo.

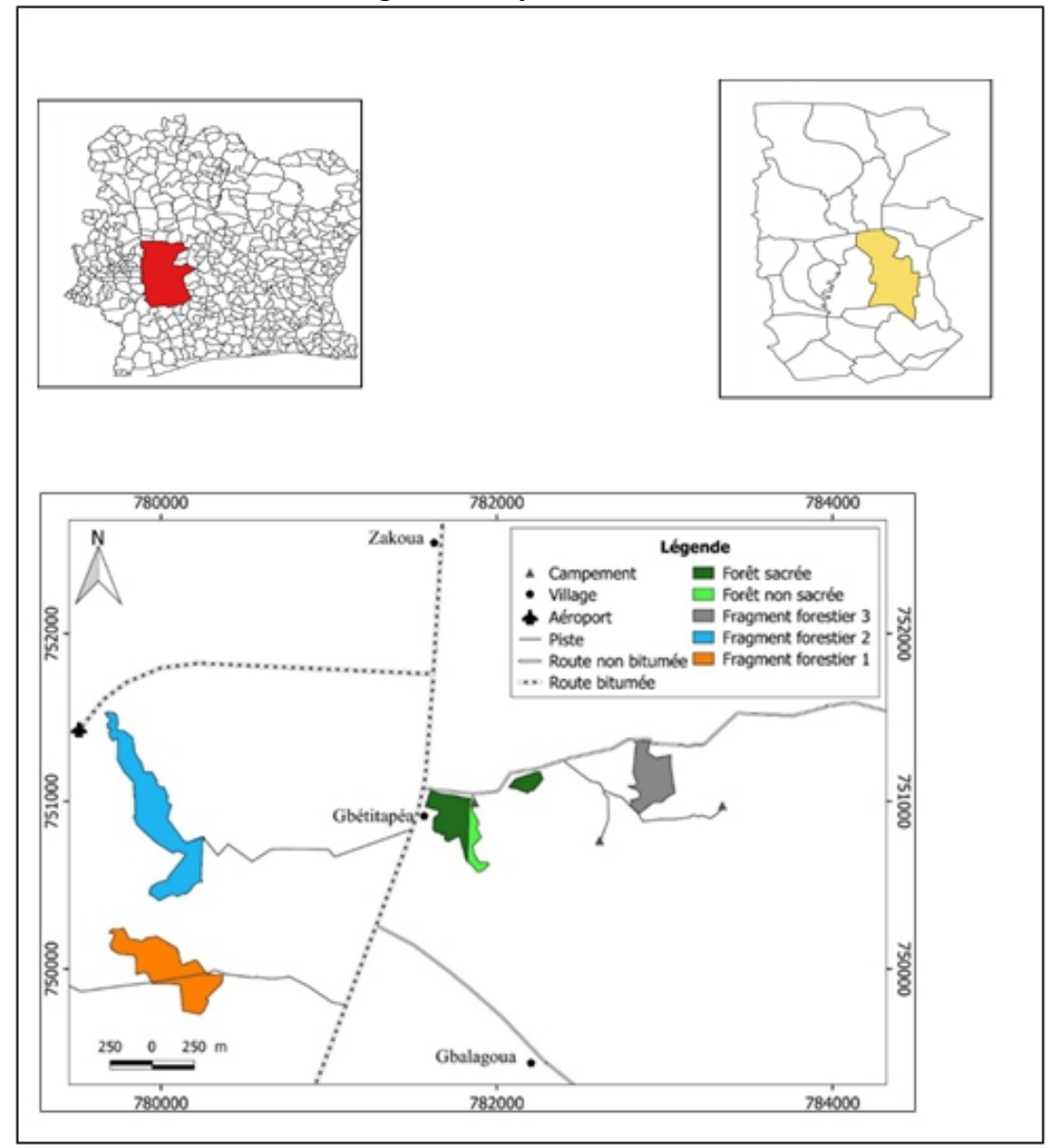

Figure 1 : Situation et localisation de la zone d'étud 


\subsection{Collecte de données}

\subsubsection{Enquêtes auprès des populations riveraines}

La méthodologie de collecte de données a consisté essentiellement en une enquête ethnozoologique dans tous les ménages des trois villages (Gbétitapéa, Zakoua et Gbalagoua). Au total, 217 ménages ont été enquêtés dont 92 à Gbétitapéa, 63 à Zakoua et 62 à Gbalagoua. Le questionnaire adressé à ces populations nous a permis de connaître la perception que les habitants ont des singes sacrés, les raisons et l'origine de la sacralisation des singes. Aussi, nous avons cherché à savoir quelles sont les relations particulières que ces habitants entretiennent avec ces singes ainsi que les raisons qui motivent ces relations. La participation aux différents rituels consacrés aux singes sacrés a constitué un volet de ce questionnaire. Concernant l'aspect économique, les questions étaient dirigées sur les bénéfices que ces populations tiraient de la gestion et de la présence de ces singes sacrés dans leur village (Gbétitapéa) et à proximité de leurs villages (Zakoua et Gbalagoua). Pour le suivi de la gestion financière des activités touristiques liées à ces singes, nous avons ouvert un cahier pour le relevé des entrées et des sorties d'argent. Dans les différents ménages les personnes prises en comptes lors de ces enquêtes, étaient constituées particulièrement de jeunes et de vieux. Nous qualifions de «Jeune » tout individu dont l'âge est compris entre 17 ans et 50 ans et de « Vieux » tout individu dont l'âge est supérieur à 50 ans (Koue, 2015).

\subsubsection{Analyse des données}

Les fréquences ont été calculées pour chaque village et ensuite pour l'ensemble des trois villages. A cet effet le logiciel SPSS 22. a été utilisé pour la construction des graphes, du calcul de $\mathrm{Chi}^{2}$, de $\mathrm{P}$ de Pearson et de V de Cramer.

Le test du Chi2 a permis de savoir si les raisons de la consommation ou non de la viande de singe et l'appartenance religieuse entretiennent une relation, avec un degré de certitude plus ou moins grand. Le V de Cramer a permis d'apprécier la force de l'association entre les variable.

\section{Résultats}

\subsection{Importance des singes au plan culturel}

\subsubsection{Perceptions locales}

Selon les informations recueillies et en considérant l'ensemble des trois villages, la majorité des enquêtés ( $\mathrm{N}=212 ; 97,69 \%)$ croit que les singes de Gbétitapéa sont issus de la transformation des aïeux lors des guerres tribales. A la demande de ces aïeux, une place à proximité du village qui était autrefois un champ de caféier leur a été accordé. Ce lieu est aujourd'hui la forêt sacrée qui abrite ces singes. Cependant, quel que soit le village, tous les 
enquêtés reconnaissent l'origine des singes à Gbétitapéa et seulement 4,84\% $(\mathrm{N}=3)$ et $3,17 \%(\mathrm{~N}=2)$ des personnes interrogées respectivement à Gbalagoua et à Zakoua estiment que ces singes viennent des forêts environnantes. Ces enquêtés qui ne reconnaissent pas l'origine des singes comme étant leurs aïeux sont essentiellement des jeunes. Aussi, l'attachement de cette population à sa culture et particulièrement aux primates non-humains se traduit par l'image de singes que portent un bâtiment culturel à Gbétitapéa (Figure 2).

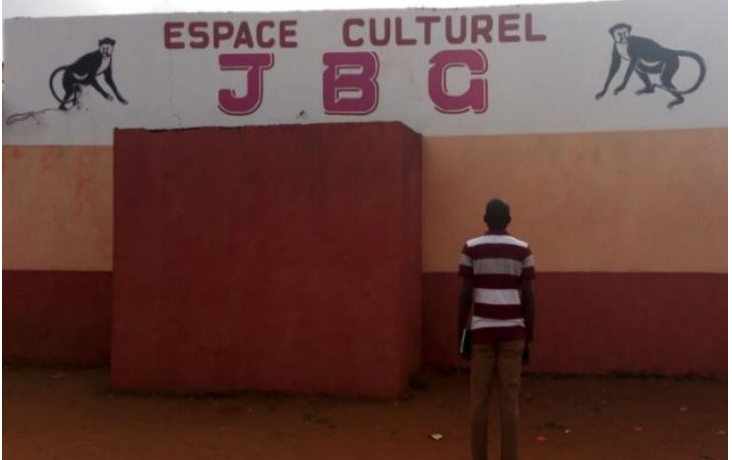

Figure 2 : Vue extérieure du bâtiment culturel du village Gbétitapéa

\subsubsection{Statut sacré et protection locale}

Tous les enquêtés, jeunes comme vieux, reconnaissent le statut sacré des singes qui vivent dans cette forêt sacré, protégés de la chasse et interdits à la consommation dans le village. Mais, en fonction du village et le foyer domestique, la viande de singe est, soit autorisée ou soit non autorisée. A Gbétitapéa, 73,91\% ( $\mathrm{N}=68)$ des enquêtés ne consomment pas la viande de singe alors que 26,09\% ( $\mathrm{N}=24)$ en consomment. A Zakoua, 52,38 \% ( $\mathrm{N}=33)$ des personnes enquêtées ne consomment pas la viande de singe contre 47,62 $\%(\mathrm{~N}=30)$ qui en consomment. A Gbalagoua, la proportion des enquêtés qui ne consomment pas la viande de singe s'élève à 9,68\%(N=6), alors que la proportion de ceux qui en consomment est de 90,32\% ( $\mathrm{N}=56)$ (figure 3$)$.

Une relation existe entre la consommation ou non de la viande de singe et les villages selon la statistique de $\mathrm{Chi}^{2}$ de Pearson $\left(\mathrm{Chi}^{2}=72,01 ; \mathrm{P}<0,001\right)$. Une telle valeur est très hautement significative. L'intensité du lien entre la consommation ou non et les villages a été évaluée en recourant à la statistique de V de Cramer qui est 0,57, soit $57 \%$. Bien que la valeur de V de cramer soit moyenne, ce lien est très hautement significatif $(\mathrm{P}<0,001)$.

La raison de la consommation ou non évoquée diffère selon les enquêtés. Pour les enquêtés de Gbétitapéa, six raisons sont évoquées par ceux qui ne consomment pas la viande de singes. Des enquêtés $(20,05 \% ; \mathrm{N}=15)$ ne consomment pas la viande de singe du fait du totem dont ils ont hérité de 
leurs parents. Certains enquêtés évoquent les raisons de ressemblance à l'Homme $(25 \%, \mathrm{~N}=17)$ et le respect de la tradition $(23,53 \% ; \mathrm{N}=16)$. L'héritage est aussi l'une des raisons de la non consommation de la viande de singe dont ceux qui l'évoquent se proclament être le garant $(16,17 \% ; \mathrm{N}=11)$. Certains enquêtés s'abstiennent de la consommation de cette viande afin de pérenniser l'attraction touristique $(10,29 \% ; \mathrm{N}=7)$ et permettre à la génération future de connaître les singes $(2,94 \% ; \mathrm{N}=2)$. Pourtant, deux raisons sont évoquées par ceux qui consomment la viande de singes. Ce sont : " considérée comme des animaux sauvages » $(75 \% ; \mathrm{N}=15)$ et «pas de totem dans la religion chrétienne » $(25 \% ; \mathrm{N}=9)$.

A Zakoua, les enquêtés qui ne consomment pas la viande de singe $(52,38 \% ; \mathrm{N}=33)$ ont aussi des raisons diverses. Les raisons évoquées par ces personnes sont la ressemble du singe à l'Homme $(66,67 \% ; \mathrm{N}=22)$, le respect de la tradition du village Gbétitapéa $(18,18 \% ; \mathrm{N}=6)$ et la conservation des singes pour les générations futures $(15,15 \% ; \mathrm{N}=5)$. Pour ceux qui consomment la viande de singe $(47,62 \% ; \mathrm{N}=30)$, pas de totem dans la religion est une des raisons évoquées $(90 \%)$. Par ailleurs, le singe est considéré comme tout autre animal sauvage pour $10 \%(\mathrm{~N}=3)$ d'individus qui consomment sa viande.

A l'instar des villages Gbétitapéa et de Zakou, il y a aussi à Gbalagoua des enquêtés $(9,68 \% ; \mathrm{N}=6)$ qui ne consomment pas la viande de brousse pour des raisons différentes. La seule raison évoquée est la conservation pour la génération future. Mais il existe également des enquêtés $(90,32 \% ; \mathrm{N}=56)$ qui consomment la viande de ces animaux. Les raisons avancées ici sont de trois sortes. Il y a des enquêtés $(58,92 \%$; $=33)$ qui ont affirmé qu'ils n'ont pas de totem dans la religion à laquelle ils appartiennent. En outre, certaines personnes $(27,79 \% ; \mathrm{N}=15)$ ont évoqué le fait que ce n'est pas leur totem. Et d'autres $(14,29 \% ; \mathrm{N}=8)$ les considèrent comme les autres animaux sauvages (Figure 4). Malgré toutes les raisons avancées relatives à la consommation de viande de singe, tous les enquêtés de Zakoua et de Gbalagoua, ainsi que 89, $13 \%(\mathrm{~N}=82)$ de la population enquêtée de Gbétitapéa sont d'accord pour un appui dans la conservation de leur forêt sacrée. 


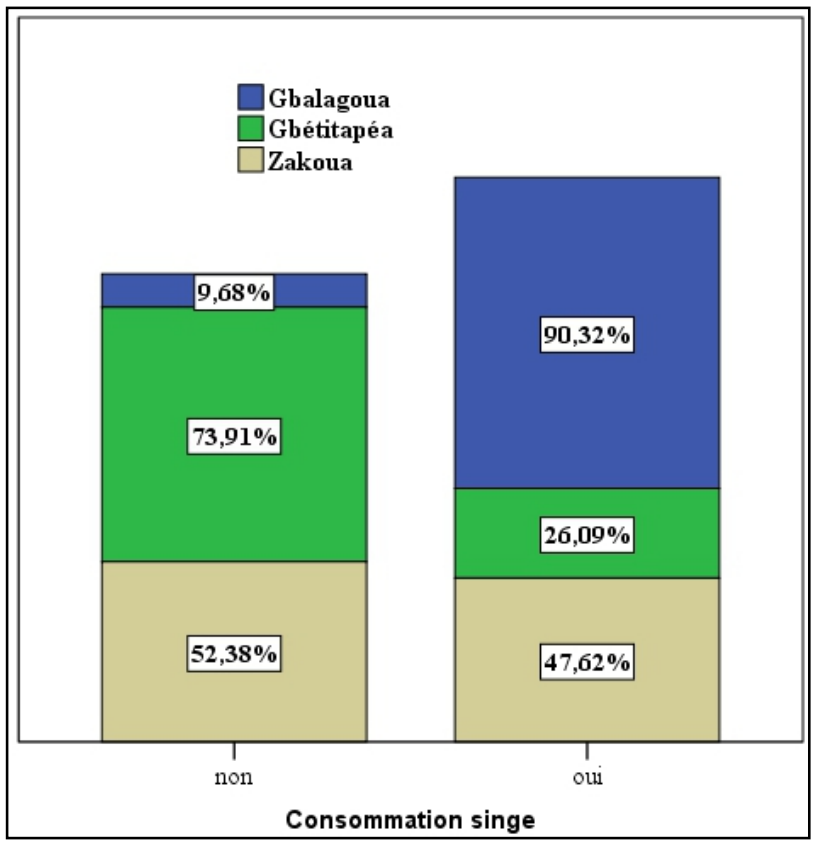

Figure 3 : Proportion des individus consommant ou non la viande de singe selon le village

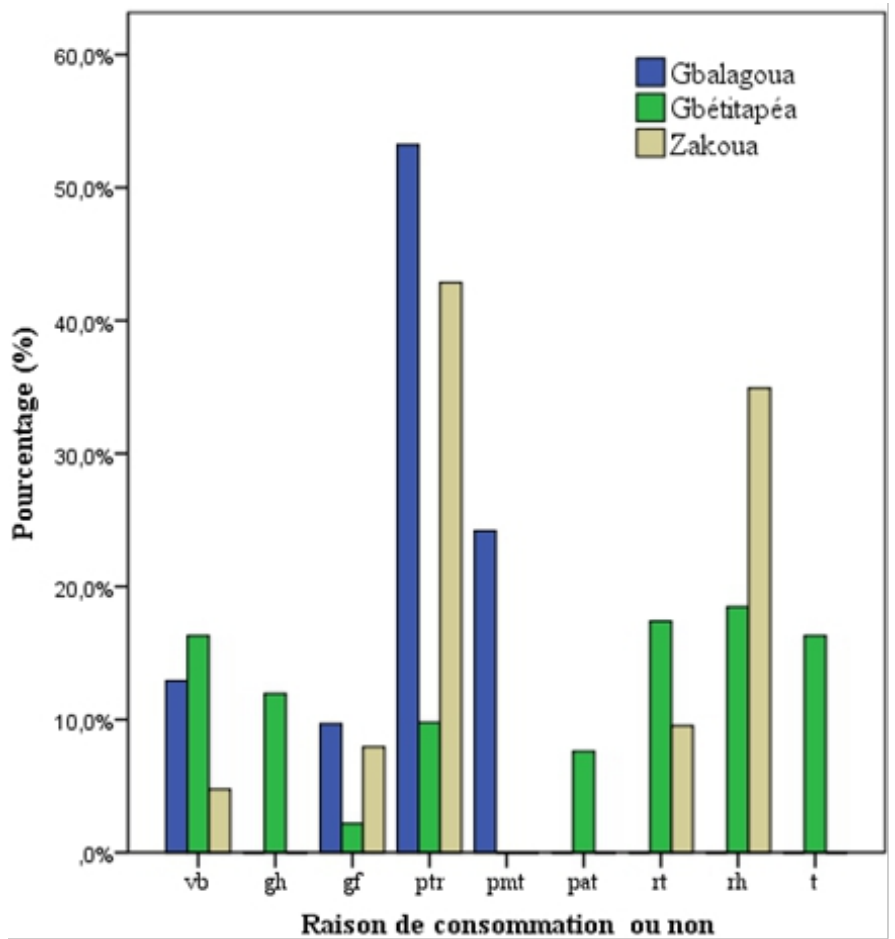

Figure 4 : Fréquence des raisons des rapports avec les singes selon le village $\mathrm{vb}=$ viande de brousse $; \mathrm{gh}=$ garant de l'héritage $; \mathrm{gf}=$ génération future $; \mathrm{ptr}=$ pas de totem dans la religion $; \mathrm{pmt}=$ pas mon totem $;$ pat $=$ pérenniser l'attraction touristique $; \mathrm{rt}=$ respect de la tradition ; $r$ h $=$ ressemble à l'homme 
La raison de la consommation ou non de la viande de singe est liée à l'appartenance religieuse des enquêtés $\left(\mathrm{Chi}^{2}=155,28 ; \mathrm{ddl}=32 ; \mathrm{P}<0,001\right)$. Cette liaison s'avère forte selon le $\mathrm{V}$ de Cramer dont la valeur est 0,623 soit $62,3 \%$. Ainsi, en fonction de l'appartenance religieuse, les raisons de la consommation ou non de viande de singe diffère. La raison la plus évoquée par les chrétiens consommant la viande de singe est qu'il n'existe « pas de totem dans la religion ». Les raisons les plus citées des chrétiens ne consommant pas la viande de ce mammifère sont « totem, pérenniser l'attraction touristique, ressemble à l'homme et respect de la tradition ». Les raisons telles que «viande de brousse » et «pas mon totem » sont plus évoquées par les musulmans, les animistes et ceux qui n'ont pas de religion (aucune) consommant la viande de singe. Les raisons « générations futures et garant de l'héritage » sont les plus citées par toux ceux ne consommant pas la viande de brousse quel que soit l'appartenance religieuse (Figure 5).

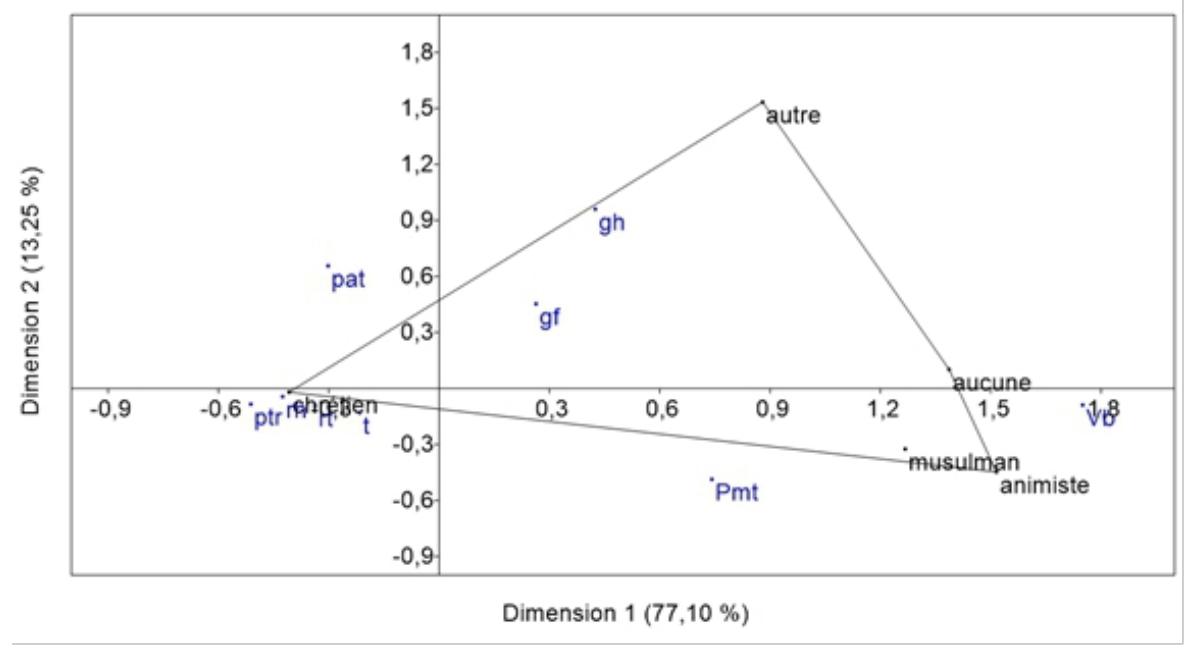

Figure 5 : Raison de la consommation ou non de la viande de singe en fonction de l'appartenance religieuse

Vb : viande de brousse; gh : garant de l'héritage; gf : génération future ; pat : péréniser l'attraction touristique; $\mathbf{p t r}$ : pas de totem dans la réligion; $\mathbf{r h}$ : ressemble à l'homme; $\mathbf{t}$ : totem ; rt : respect de la tradition ; pmt : pas mon totem

\subsubsection{Relations hommes-singes et la pérennité de la protection traditionnelle}

La majorité des enquêtés $(90,22 \%)$ de Gbétitapéa déclarent n'avoir pas de relations particulières avec les singes, ils ne s'intéressent donc pas aux rituels qui leurs sont associés. Par contre une minorité (9,78 \%) affirment avoir des relations particulières avec les singes. Selon les investigations menées dans les villages voisins (Zakou et Gbalagoua), les rituels consacrés aux singes 
reviennent à la population de Gbétitapéa. Dans cette population qui participe au rituel des singes, $66,67 \%$ sont des vieux et 33,33\% sont des jeunes. Les individus de cette population ne sont ni chrétiens, ni musulmans mais sont soit animistes, soit ils appartiennent à une autre religion ou aucune.

Trois raisons principales sont les causes de l'abandon et du désintérêt du rituel consacré aux singes. Parmi ces raisons, l'invasion des religions étrangères a été le plus cité (77,17\%). La modernisation est aussi l'une des raisons de l'abandon des rituels consacrés aux singes avec un taux de citation de 22,83 $\%$. L'école a été aussi mentionnée comme étant l'une des raisons avec une fréquence de citation de $11,96 \%$.

\subsubsection{Estimation de la taille des populations de singe}

La taille des groupes de singes rencontrée avant dans les fragments de forêt à Gbétitapéa a été estimée entre 25 et 100 individus avant les années 2000 par la population enquêtée. Alors que maintenant cette taille connait une diminution et a été estimée entre 15 et 40 individus.

Les raisons de cette diminution ont été évoquées par les populations enquêtés. A cet effet, quatre (4) raisons ont été révélées par les enquêtés. La chasse est l'une de ces raisons dont la fréquence de mention s'élève à 65,44\% $(\mathrm{N}=142)$. Le manque de nourriture a été aussi évoqué comme étant l'une des raisons de la diminution de la population des singes $(16,13 \% ; \mathrm{N}=35)$. Le non-respect de la tradition est l'une des causes de cette diminution (11,06\% ; $\mathrm{N}=24)$. Aussi, la réduction de l'habitat de ces animaux a été reconnue par 7,37 $\%(\mathrm{~N}=16)$ des enquêté qui ont affirmé qu'elle contribuait à la diminution de la population des singes (Figure 6).

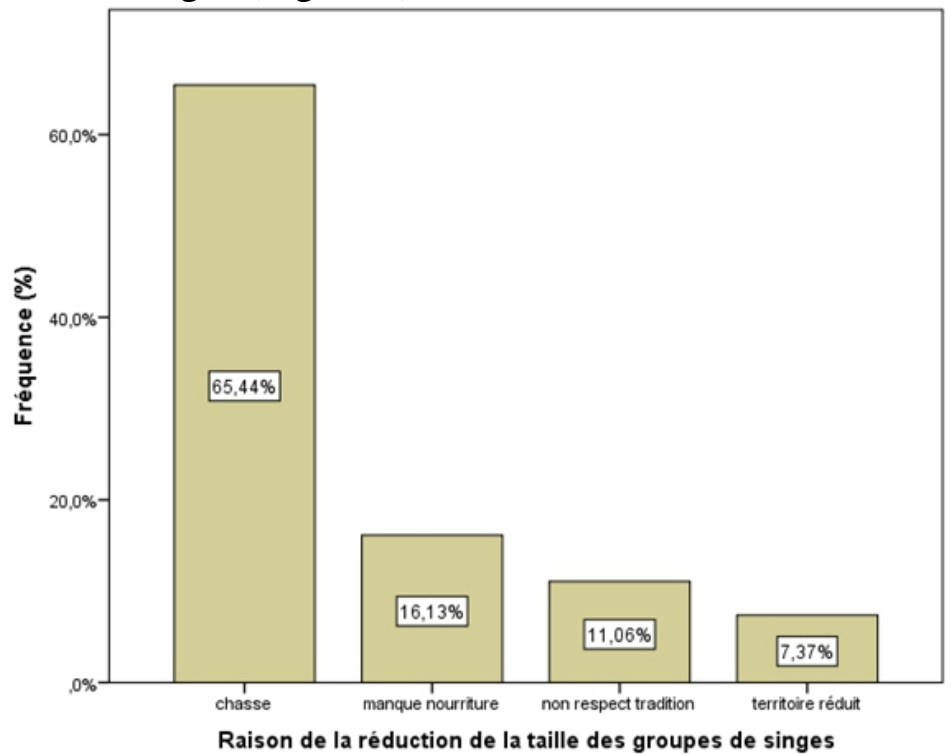

Figure 6 : Fréquence des raisons de réduction de la taille des groupes de singes 


\subsection{Importance économique}

Le seul fragment forestier bien conservé (forêt sacrée) constitue une source d'activité économique. La présence des singes et leur cohabitation avec la population attirent quelques scientifiques et curieux qui en font un lieu touristique (Figure 7). Economiquement ce site rapporte au village selon la population. Le nombre de visite de ce site varie selon le mois. Ainsi, pendant notre étude, les mois de janvier, mars, juin et septembre n'ont pas enregistré de visites. Les autres mois connaissent des visites ainsi que des recettes, mais dans des proportions différentes. Le nombre de visites n'est pas proportionnel aux recettes. Le mois de novembre enregistre le plus grand taux de visites $(21,74 \%)$ dans l'année avec la plus forte recette (57000 Fcfa). Avril et décembre sont des mois qui connaissent des visiteurs (17,39\%) avec respectivement des recettes de $27000 \mathrm{Fcfa}$ et $22000 \mathrm{Fcfa}$. Les taux de visites dans les mois de février et juillet sont de 13,04 \% des visites annuelles dont les recettes sont respectivement $25000 \mathrm{Fcfa}$ et $12000 \mathrm{Fcfa}$. Le mois août enregistre $8,7 \%$ des visites annuelles avec un montant de 7000 Fcfa. Mai et octobre enregistrent $4,35 \%$ des visites annuelles avec des recettes respectivement 12000 et 5000 Fcfa (Figure 8).

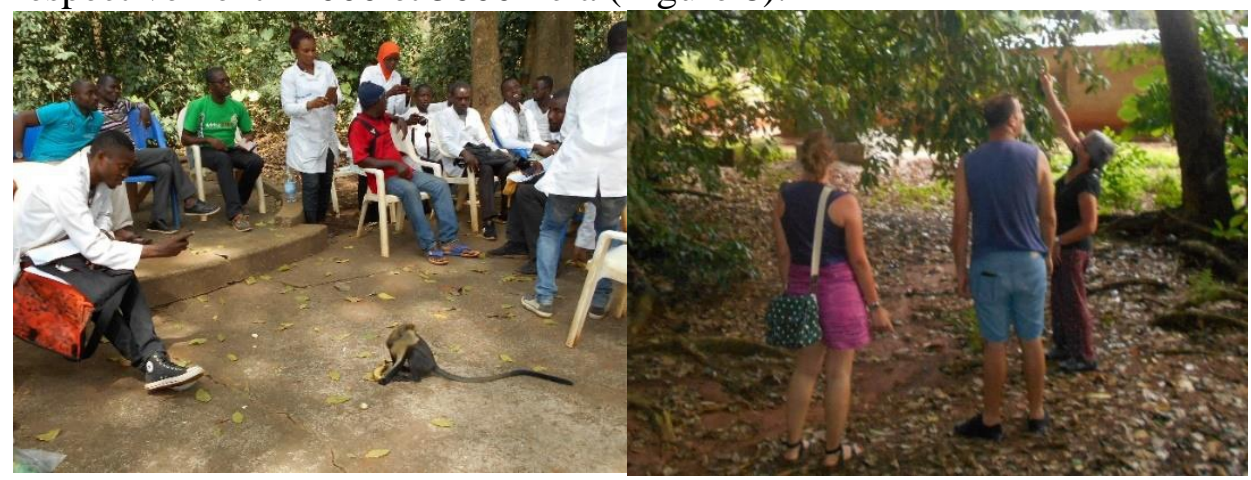

a. Etude scientifique des étudiants

b. Visite touristique d'une famille

Figure 7 ( $a$ et b) : Visiteurs des singes sacrés de Gbétitapéa

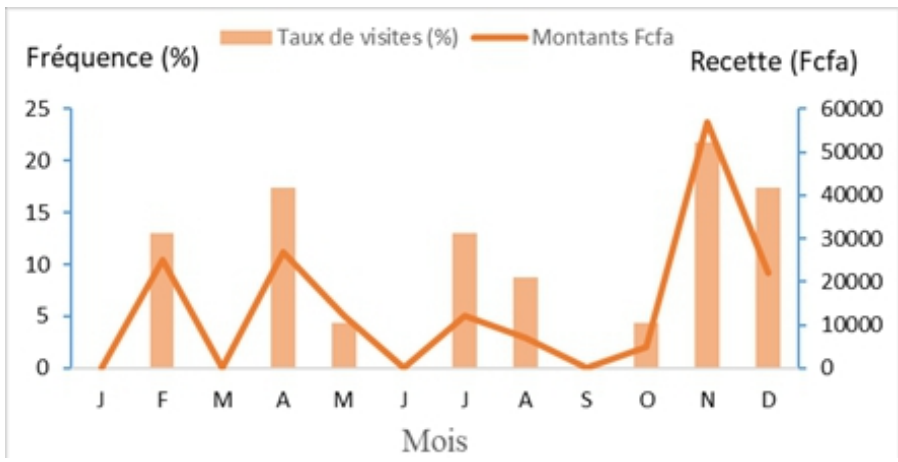

Figure 8 : Proportion des visites et des recettes perçus selon le mois Fcfa : Franc de la communauté financière africaine 
Certaines activités présentant aussi un intérêt économique voient le jour. Ainsi, l'installation dans le village d'une buvette à l'effigie du singe (Figure 9) traduit l'attachement aux singes et tentent d'en faire un élément de l'économie locale. Cependant tous les enquêtés de Gbétitapéa manifestent leur mécontentement vis-à-vis de de la gestion de la forêt sacrée du fait qu'elle revient uniquement au gardien de la forêt.

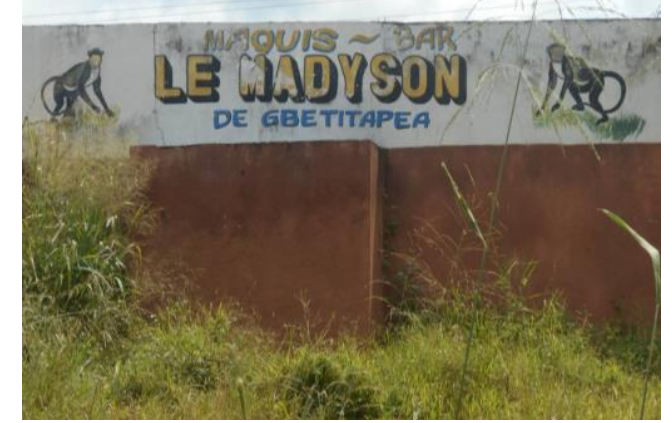

Figure 9 : Vue de l'extérieur d'une buvette portant les images de singes

\subsection{Discussion}

Notre étude révèle que les singes de Gbétitapéa représentent pour la population une valeur traditionnelle dont les villageois de toutes catégories (les autochtones, les allochtones et les étrangers) connaissent bien son importance. Les singes incarnent les aïeux, et au-delà des frontières, les villages voisins semblent avoir conscience de l'intérêt accordé aux singes. La connaissance traditionnelle se serait transmise de génération en génération à travers un savoir symbolique portant sur les représentations de ces entités (Sperber, 1974).

Les traditions africaines ont toujours considéré la nature et ses ressources comme des éléments sacrés et revêtus d'une nature divine. Cette sacralisation englobe un ensemble de savoirs naturalistes pouvant concourir à la conservation et l'utilisation durable de la diversité biologique (Friedberg, 1986 ; Barreau, 1993).

Les croyances évoquées à Gbétitapéa et relatives au statut sacré des singes sont partagées par toute la population. Cette conception totémique où les singes incarnent des personnes sont similaires des tabous traditionnels observés dans les villages Boabeng et Fiema dans la région de Brong Ahafo au Ghana où le colobe de Geoffroy et le Mone de Campbell vivent en parfaite harmonie avec les habitants (Akowuah et al., 1975 ; Kankam, 1997 ; Saj et al., 2006). Mêmes si des rituels similaires sont pratiqués, ici le tabou vient du fait que ces singes sont considérés comme leurs parents décédés qui reviennent vivre sous forme animal avec eux au village. De même, dans le village de Soko (région de Bondoukou) au nord-est d'Abidjan à $3 \mathrm{~km}$ de la frontière ghanéenne, les espèces de singes Chlorocebus tantalus et Erythrocebus patas 
vivent en parfaite harmonie avec les habitants et sont interdits de chasse (Ibo, 1999) comme l'exige la coutume qui voient ces singes comme leurs parents directs. Cependant cette croyance n'empêche pas que ces animaux soient victimes de consommation de leur viande.

La viande de singes fait partie des produits de consommation dans certains foyers pendant que d'autres restent fidèles à leur croyance. Il existe aussi une grande affinité entre la consommation ou non de viande de singes et le village. Le lien très fort entre la consommation ou non de la viande de singe et le village pourrait venir du fait que chaque village aurait ses propres interdits et ne partage pas vraiment les interdits des autres. En effet, la sacralisation de la nature par les communautés au sein des forêts découle de diverses significations qui sont le reflet de leurs traditions. Les sociétés africaines reposent sur une philosophie de la négociation avec la nature. Cette philosophie révèle l'intérêt des forêts sacrées et celui des pratiques totémiques dans la gestion de la nature. Ainsi les systèmes de gestion varient en fonction des peuples et des groupes ethniques (Kragbe et Tahou, 2010 ; Djego-Djossou et al., 2012). Mais, toujours est-il que le peuple concerné par la sacralisation reste généralement fidèle à ses croyances.

La différence de consommateurs de viande de singes dans les trois villages montre combien la population de Gbétitapéa reste encore attachée à ses coutumes. Cette importance se limite à la frontière du village qui serait donc responsable du nombre élevé de consommateurs dans les villages voisins. En effet, les peuples africains se réfèrent souvent à des divinités locales ou individuelles (Kragbe et Tahoux, 2010). C'est ainsi que les raisons de la consommation ou non des singes diffère selon les personnes enquêtées.

Les raisons de la non consommation de la viande de singe vont toutes dans le sens de la protection des singes. Quel que soit la raison, il s'agit dans tous les cas d'interdiction totémique. Ces totems soulignent le rôle important des communautés locales dans la mise en place d'une gestion durable des écosystèmes naturels (Kragbe et Tahoux, 2010). Ces raisons sont similaires à la non consommation de viande sacrée dans certaines régions d'Afrique (Kpera, 2002 ; Saj et al., 2006 ; Djego-Djossou et al., 2012). En revanche, les raisons de la consommation de la viande de singe et de l'abandon des rituels consacrés aux singes seraient dues au passage des colons et de l'expansion des religions étrangères. En effet la colonisation française en Côte d'Ivoire a abouti à une exploitation vertigineuse et anarchique des ressources naturelles non seulement du fait de la recherche du profit économique, mais également à cause de la pression démographique en milieu rural. Le colonisateur se proclame aussi laïc et ignore les croyances locales, laissant ainsi à chaque individu ou groupe d'individus la liberté d'adhérer à la religion de son choix dans une société multiconfessionnelle. Cette situation crée un monde nouveau dans lequel les anciennes conceptions et les vieilles institutions peuvent perdre 
leur signification et leur raison d'être. Les données naturelles et historiques sont en passe de se trouver révoquées et un nouvel ordre des choses remplace l'ancien (Nené Bi, 2005). En outre les bouleversements politiques, sociaux et économiques entraînent une mutation dans les coutumes et les pratiques religieuses locales. Aujourd'hui, les communautés ivoiriennes, à l'image de toutes les communautés traditionnelles africaines, sont le lieu de nombreuses mutations socioculturelles dont la plupart constitue des facteurs d'abandon ou de rejet des coutumes anciennes. Ainsi, la pression démographique, la raréfaction des terres et l'expansion des religions monothéistes (Yamoi et al.,) ont constitué des obstacles majeurs au fonctionnement harmonieux du système traditionnel de gestion de la nature (Juhé-Beaulaton, 2008 ; Kragbe et Tahoux, 2010). Le dysfonctionnement de ces systèmes traditionnels va impacter négativement la biodiversité en général et les espèces de singes en particulier.

Les communautés locales constatent la baisse des effectifs des groupes de singes dans les fragments de forêt. Selon les raisons qu'elles ont évoquées (la chasse, la réduction des habitats, la chasse et le non-respect des traditions), les populations locales sembleraient être conscientes des pratiques qui dégradent l'environnement. Ces raisons évoquées sont confirmées dans les travaux de Alexandre (1992), Bakayoko et al., (2001), Caspary et al., (2001), Koné (2004), Bitty et al., (2013) et Yéo et al., (2013). Malgré la perte de ces espèces animales, les populations tentent tant bien que mal de profiter de la situation dégradante.

Malgré les menaces anthropiques, la forêt et ses singes sacrés constituent un véritable site d'attraction touristique à Gbétitapéa. Ainsi, la valeur culturelle de la forêt sacrée vire sensiblement vers une valeur économique sans précédent. Les recettes que rapporte cet écotourisme de la forêt sacrée de Gbétitapéa et ses singes semi-domestiqués montrent l'importance de ce site pour les populations locales. En effet la culture protectionniste en faveur des singes a permis une cohabitation pacifique entre hommes et singes depuis plusieurs décennies. Cette cohabitation a favorisé l'habituation des singes et fait de la forêt sacrée un endroit où les singes peuvent être facilement observés en milieu naturel. C'est donc un site idéal pour l'étude éco-éthologique des primates non-humains. (Djego-Djossou et al., 2012).

\section{Conclusion}

La sacralisation de l'environnement basée sur des ressources naturelles remonte toujours aux fondements culturels du terroir local. Elle est, de ce fait, l'expression d'une symbiose dialectique entre la société traditionnelle et le milieu naturel. C'est dans ce cadre qu'il convient de souligner l'importance que revêt l'usage coutumier des ressources de l'environnement pour la population de Gbétitapéa. Cette pratique endogène nous permet de dire que la 
population de Gbétitapéa dispose dans son organisation sociale, de principes de développement durable dans ses rapports avec la nature. En outre, les principes traditionnels étant transmis de générations en générations, la majorité des populations connait l'origine sacrée des singes. En dépit de cette connaissance une minorité de la population de Gbétitapéa et la majorité de celle des villages voisins consomment la viande de singe. Aussi, l'écotourisme se développant sur ce site sacré, constitue-t-il une ressource économique importante bien qu'encore traditionnelle et connaissant des problèmes organisationnels. Du point de vue de la protection de l'environnement, la protection traditionnelle semblerait plus authentique, car celle-ci, par son essence est postérieure à l'action de la transformation du milieu. C'est pourquoi, il serait nécessaire de diriger les recherches sur l'origine, les buts et surtout la place des lieux sacrés des sociétés traditionnelles afin de connaître leur importance socio-culturelle et économique.

\section{References:}

1. Adjakpa, J.B. et Tchabi, A. (2002). Contribution à l'inventaire des oiseaux sauvages utilisés comme matières premières en pharmacopée traditionnelle au Bénin, Nature et Faune vol 18, 1, pp. 14-22.

2. Akowuah, D. K., Rice K., Merz A. et Sackey V.A. (1975). The children of gods. Journal Ghana Wildlife Society 1(2): 19-22.

3. Alexandre, D.Y. (1992). La survie des forêts tropicales. La Recherche 244 (23): 693 - 694.

4. Asibey, E.O.A. (1977). Experted Effects of Land-Use Patterns on Future Supplies of Bushmeat in Africa South of the Sahara. Envi.Cons, 4: 43-49.

5. Avocèvou, C., Sinsin, B., Adégbidi A., Dossou, G. et Van Damme, P. (2009). Sustainable use of non-timber products: Impact of fruit harvesting on Pentadesma butyracea regeneration and financial analysis of its products trade in Benin. In Forest Ecology and Management 257 (2009) 1930-1938.

6. Awono, A. et N'doye, O. (2003). Les vertus des PFNL dans la zone forestière humide du Cameroun et l'impact de leur exploitation sur l'environnement. In WFC - XII- 0710 A2.

7. Bakayoko, A., Kouamé, N.F., Tra, Bi H.F. et Traoré, D. (2001). Quelques aspects floristiques et structuraux de la forest classée de Bossématié, dans 1'Est de la Côte d 'Ivoire = Some strutural and floristic aspects of Bossematié forest in the East of Côte d'Ivoire. Annales de Botanique de l'Afrique de l'Ouest 0(0): 7-19. 
8. Barreau, H. (1993). La construction de la notion du temps, (t. 1, 2, 3), Thèse de Doctorat d'Etat, Atelier d'impression du Département de Physique, Université Louis Pasteur, Strasbourg, 1478 p.

9. Béné, J.C.K. (2007). Répertoire et contextes sociaux des cris unitaires du colobe vert (Procobus verus) dans le Parc National de Taï (PNT), Côte d'Ivoire, 4(2) : 137-147.

10. Bhagwat, S.A. et Rutte, C. (2006). Sacred groves : potential for biodiversity management, Front Ecol. Environ, 4, 10, pp 519-524.

11. Bitty, A.E., Kadjo, B., Gonedele bi, O.O.M. et Kouassi, K.K. (2013). Inventaire de la faune mammalogique d'une forêt urbaine, le Parc National du Banco, Côte d'Ivoire. Int. J. Biol. Chem. Sci. 7(4): 16781687, August 2013, 10p.

12. Brou, Y T. (2005). Climat, mutations socio- économiques et paysages en Côte d'Ivoire. Mémoire de synthèse des activités scientifiques présenté en vue de l'obtention de l'habilitation à Diriger des Recherches. Université des Sciences et Technologies de Lille, France, 212p.

13. Butare, I. (2001). Les pratiques culturelles. Sauvegarde et conservation de la biodiversité en Afrique de l'Ouest et du Centre : Actes du Séminaire-Atelier de Ouagadougou (Burkina Faso), du 18 au 21 juin 2001, CRDI, Canada, 251 p.

14. Caspary, H.U. (1999). Wildlife utilization in Côte d'Ivoire and West Africa - potentials and contraints for development cooperation. GTZ, Eschborn, 147 p.

15. Caspary, H.U. Koné, I., Prout, C. et De Pauw, M. (2001). La chasse et la filière de viande de brousse dans l'espace Taï, Côte d'Ivoire. Tropenbos - Côte d'Ivoire série 2, 98 p.

16. Codjia, J.T.C., Vihotogbe, R., Assogbadjo, A.E. et Biaou, G. (2009). Le marché du crincrin (Corchorus tridens) au Bénin. Annales des sciences agronomiques (FSA-UAC, Bénin), 19 p.

17. Dadjo, C. (2011). Caractérisation ethnobotanique, morphologique et spatiale de Vitex doniana Sweet (Verbenaceae) au Sud-Bénin ; Thèse d'Ingénieur Agronome; Faculté des Sciences Agronomiques de l'Université d'Abomey-Calavi, Bénin, 86 p.

18. Deguenonvo, M.N. (2011). Evaluation écologique et socioéconomique de Mondia whitei (hook.f.) Skeels (asclepiadaceae) au Sud-benin. Thèse d'ingénieur agronome, FSA/UAC, 64p.

19. Djego-Djossou, S., Huynen, M.C., Djego, J. et Sinsin, B. (2012). « Croyances Traditionnelles et Conservation du Colobe de Geoffroy, Colobus vellerosus (Geoffroy, 1834), dans la Forêt Sacrée de Kikélé, Bénin (Afrique de l'Ouest) », African Primates 7 (2): 193-202 
20. Fandohan, B., Assogbadjo, A.E., Glèlè Kakai, R.G., Kyndt, T., De Caluwe, E., Codjia, J.T.C. et Sinsin, B. (2010). Women's Traditional Knowledge, Use Value and the Contribution of Tamarind (Tamarindus indica L.) to Rural Household's Cash Income in Benin. Economic Botany, 64(3), 2010, pp. 248-259.

21. FFEM (2007). Conservation et valorisation de la forêt sacrée de Kpinkonzoumé et de l'îlot forestier de Houanvè. https://uicn.fr/wpcontent/uploads/2017/02/cerget-fiche-communication-ffem-1 [consulté le 04/07/2019]

22. Flores, C.F. et Ashton, P.M.S. (2000). Harvesting impact and economic value of Geonoma deversa, arecaceae, an understory palm used for roof thatching in the Peruvian Amazon. Economic Botany, 54 (3), 267-277.

23. Friedberg, C. (1986). « Les savoirs populaires sur la nature (Les hommes et le milieu naturel) », Revue d'ethnologie de l'Europe, $\mathrm{n}^{\circ} 6$, 313-336, Mémoires du Muséum national d'histoire naturelle, série botanique. Paris.

24. Gami, N. (1999). Etude ethnozoologique dans et autour de la réserve de Conkouati (village N'tié-tié de Cotovindou). Rapport final UICN, PROGECAP/GEF, Congo Brazzaville.

25. Gomé, G. H., 2003, Forêts sacrées de Côte d'Ivoire : la tradition au secours de l'environnement, pp 33 - 45, Butare, I., Les pratiques culturelles. Sauvegarde et conservation de la biodiversité en Afrique de l'Ouest et du Centre : Actes du Séminaire-Atelier de Ouagadougou (Burkina Faso), du 18 au 21 juin 2001, CRDI, Canada, 251 p.

26. Goussanou, A.C., Tente, B., Djègo, J., Agbani, P. et Sinsin, B. (2011). Inventaire, caractérisation et mode de gestion de quelques produits forestiers non ligneux dans le bassin versant de la Donga. In Annales des Sciences Agronomiques ; Bénin 14 (1) 77-99, 2011.

27. Gouwakinnou, G.N., Lykke, A.M., Assogbadjo, A.E. et Sinsin, B. (2011). Local knowledge, pattern and diversity of use of Sclerocarya birrea. Journal of Ethnobiology and Ethnomedicine, 7(8): 1746-4269.

28. Ibo, J., (1999). La gestion coutumière de l'environnement en Côted'Ivoire. Bulletin du GIDIS-CI 17: 6-36.

29. Juhé-Beaulaton, D., (2008). "Sacred forests and the global challenge of biodiversity conservation: the case of Benin and Togo". Journal for the Study of Religion, Nature, and Culture, pp. 351-372.

30. Juste, J., Fa, J.E., Perez Del Va,1 J. et Castroviejo, J. (1995). Market Dynamics of Bushmeat Species in Equatorial Guinea Journal of Applied Ecology Vol. 32, No. 3 (Aug., 1995), pp. 454-467.

31. Kankam, B.O. (1997). The Population of Black and White Colobus (Colobus polykomos) and Mona Monkeys (Cercopithecus mona) at the 
Boabeng-Fiema Monkey Sanctuary and Surrounding Villages, B.Sc. Thesis, University of Science and Technology, Kumasi, Ghana. 100 p. 32. King, S. (1994). Utilization of Wildlife in Bakossiland, West cameroun with particular reference to primates. TRAFIFIC Bulletin, 14: 63-73.

33. Koffie, B.C.Y. et Kra, K.S. (2013). La région du Haut-Sassandra dans la distribution des produits vivriers agricoles en Côte d'Ivoire. Institut de Géographie Tropical, Université Félix Houphouët-Boigny de Cocody / Abidjan / Côte d'Ivoire ; Tropicale et d'Environnement, $n^{\circ}$ 2, $2013 ; 9 \mathrm{p}$.

34. Kolongo, T.S.D., Decocq, G., Yao C., Blom, E.C. et Rompaey, R.S.A., (2006). Plant species diversity in the southern part of the Tai National Park (Côte d'Ivoire). Biodiversity and Conservation, 15: 2123-2142.

35. Koné, I. (2004). Effet du braconnage sur quelques aspects du comportement du colobe bai Procolobus [piliocolobus] badius et du cercopitèque diane Cercopithecus diana diana dans le Parc National de Taï, Côte d'Ivoire, Thèse de Doctorat, Université de Cocody, Abidjan, $146 \mathrm{p}$.

36. Kouakou, C.V., Béné, J.C.K., Kouamé, N.A., Yao, K.C. \& Bamba, K. (2017). Diversity, distribution and social structure of monkey species in forest fragments of Gbetitapea, central-western Ivory Coast. Journal of Chemical, Biology an Physical Section B, 8(1) : 127-143.

37. Kouakou, Y. V., Zadou, A.D., Jean, C.N., Ouattara, K., Kouassi, S., Koffi, B.J-.C.K., Koné, I. \& Ibo, J. (2017). Perception contrastée des singes sacrés à Gbétitapéa (Centre-ouest Côte d'Ivoire). Annales de l'Université de Lomé, Série Lettres et Sciences Humaines, Tome XXXVII-1, Juin 2017, 27-38.

38. Kouame, B., Kone, D. et Yoro, G. R. (2006). La pluviométrie en 2005 et 2006 dans la moitié Sud de la Côte d'Ivoire. Bulletin le CNRA en 2006, document technique : 12-13.

39. Kpera, G. N. (2002). Impact des aménagements d'hydraulique pastorale et des mares sur la reconstitution des populations de crocodiles dans les communes de Nikki, KALALE, Ségbana, Kandi, Banikoara, Kérou, Ouassa-Péhunco et Sinendé. Thèse d'ingénieur agronome, FSA/UAC, Bénin, $102 \mathrm{p}$.

40. Kpéra, G.N., Sinsin, B. et Mensah, G.A. (2007). Mesures de conservation endogènes de la faune sauvage : Cas descrocodiles au Bénin. In First Regional Workshop on the Management of Protected Areas in West Africa. Parakou, Bénin. pp. 405-414.

41. Kragbe, A.G. et Tahoux Touao, M., (2010). La sacralisation de la nature dans la gestion de l'environnement, Revue des Sciences Sociales, $n^{\circ} 43,130-141$. 
42. Malan, D.F. Aké, A. L., Tra Bi, F. H. et Neuba, D. (2007). Diversité floristique du Parc National des Îles Éhotilé (littoral est de la Côte d'Ivoire), Bois et Forêts des Tropiques, 292, 2, pp 49-58.

43. Malan, D.F. (2009). Religion traditionnelle et gestion durable des ressources floristiques en Côte d'Ivoire : Le cas des Éhotilé, riverains du Parc National des Îles Éhotilé, [VertigO] - la revue électronique en sciences de l'environnement, 9, 2, [En ligne], URL : http://vertigo.revues.org/index8661.html. Consulté le 06 octobre 2018.

44. Myers, M., Mittermeier, R.A., Mittermeier, C.G., Da Fonseca, G.A. et Kent, J. (2000). Biodiversity hotspots for conservation priorities. Nature, 403: 853-858.

45. Ndotam, I. (2005). Gestion des aires protégées au Tchad : contraintes et perspectives pour une gestion participative, Mémoire de DESS, Université de Kinshasa, 102 pages.

46. Nene Bi, B.S. (2005). La terre et les institutions traditionnelles africaines : cas des Gouro de Côte d'Ivoire, Thèse unique de Doctorat en Droit, Université de Cocody, Abidjan, 750 p.

47. Nikolaus, G., (2001). Bird exploitation for traditional medecin in Nigeria. Malimbus, 23, pp. 45-55.

48. Norbert, N.K., François, K.N., Hauverset, A.N., Pierre, W.N. et Yao, T. (2015). Variations saisonnières des populations de mirides du cacaoyer dans la région du Haut Sassandra en Côte d'Ivoire. Journal of Animal \& Plant Sciences, Vol.25, Issue 1: 3787-3798.

49. Ouattara, T.F. (1988). La mémoire Sénoufo : bois sacrés, éducation et chefferie. Paris, Association Arsan, 175 p.

50. Posey, D. (1995). Indigenous People and Traditional Resource Right : a basic for equitable relationships ? Green College for Environmental Policy and Understanding. Oxford University Press, New York.

51. Quin, W., Ming-Xing, H., Ying, X., Xin-Shen, Z. et Fang, C. (2005). Expression of a ribosome inactivating protein (curcin 2) in Jatropha curcas is induced by stress. Journal of Biosciences, 30: 351-357.

52. Saj, T., Mather, C. et Sicotte, P. (2006). Traditional taboos in biological conservation: the case of Colobus vellerosus at BoebengFiema Monkey Sanctuary, Central Ghana. Social Science Information 45: 285-310.

53. Sie, K. et Ibo, G.J., (1990). Histoire écologique du pays Abronkulango. Bibliothèque ORSTM p 69.

54. Sperber, D. (1974). Le symbolisme en général, Paris, Hermann, 163p.

55. UICN (2008). Sites naturels sacrés. Lignes directrices pour les gestionnaires d'aires protégées, (Wild R. et McLeod C. (éds), n 16 p 106. 
56. Williams, V.L., Cunningham, A.B., Kemp, A.C. et Bruyns, R.K. (2014). Risks to Birds Traded for African Traditional Medicine: A Quantitative Assessment. PLoS ONE, 9, 8, 15 p.

57. Yamoi, V.K., Didié, A.Z., Jean, C. N., Karim, O., Siméon, K., Béné, J.C.K. et Inza, K., Jonas, I. (2017). Perception contrastée des singes sacrés à Gbétitapéa (centre-ouest Cote d'Ivoire) Ann.Univ. de Lomé, Sér.Lett., Tome XXXVII-1, Juin 2017, pp.27-38.

58. Yéo, K., Tiho, S., Ouattara, K., Konate, S., Kouakou, L.M.M. et Fofana, M. (2013). Impact de la fragmentation et de la pression humaine sur la relique forestière de l'Université d'Abobo-Adjamé (Côte d'Ivoire). Journal of Applied Biosciences, 61, pp. 4551- 4565.

59. Zadou, A. D., Koné, I., Vincent Kouassi Mouroufie, K. V., Constant Adou, Y. Y. C., Gleanou, K. E, Kablan, A. Y., Coulibaly, D. and Guéhilbo, J. (2011). Valeur de la forêt des Marais Tanoé-Ehy (sud-est de la Côte d'Ioire) pour la conservation : dimension socioanthropologique. Tropical Conservation Science Vol.4 (4):373385,2011 . 